\title{
Das Augsburger Domkapitel: Vermögensverwaltung zwischen Ritter- und Gelehrtenkultur im 15. Jahrhundert
}

\section{Mitwirkung des Domkapitels an bischöflichen Reformmaßnahmen?}

Die Domkapitel des 15. Jahrhunderts, weder in Augsburg noch anderenorts, waren dem Forschungsstand nach keine Zentren und Anstoßgeber der Kirchen- und Klosterreformbewegungen. Doch kann deren Geschichte nicht isoliert von Domkapiteln betrachtet werden, nicht nur weil die Domkapitel Anlass für Reformdiskussionen boten, sondern weil sie in der Verwaltung der Diözesen eine zentrale Rolle spielten, die von Initiatoren der Reform nicht ignoriert werden konnte. ${ }^{1}$ Insofern muss von einer Vernetzung von Domkapitels- und Kirchenreformgeschichte ausgegangen werden.

Weitereichende persönliche Verflechtungen spätmittelalterlicher Domkapitel und ihre Konsequenzen sind vor allem durch Monographien zu Speyer, Mainz, Trier und Konstanz in den Blick der neueren Forschung gelangt. ${ }^{2}$ Nach Andreas Bihrer bildete das Konstanzer Domkapitel den inneren Kern eines Bischofshofes, der seinerseits in sozial gut vernetzte Hofparteien untergliedert werden kann. ${ }^{3}$ Als Bischofswähler und Pfründenbesitzer waren die Domkapitulare hier die wichtigsten „Träger von Kontinuität“. ${ }^{4}$

1 Dies zeigt besonders auch der Beitrag von Werner Williams-Krapp in diesem Band. Zu der von ihm thematisierten Prädikatur am Augsburger Dom wird am Ende dieses Beitrages die Stiftungsurkunde vorgestellt und abgebildet.

2 Holbach 1987; Hollmann 1990; Bihrer 2005.

3 Die Forschungen von Bihrer beziehen sich auf das 14. Jahrhundert, die Ergebnisse dürften sinngemäß aber auch für das 15. Jahrhundert Bestand haben. Zum Konstanzer Bischofshofs des 14. Jahrhunderts formulierte Bihrer zuletzt: „Eine besondere Rolle unter den Pfründinhabern spielten die Domherren, die nicht nur Ämter in der bischöflichen Verwaltung besetzten oder einige von diesen sogar ausschließlich für sich beanspruchten, sondern die auch als Wahlmänner, aufgrund einer möglichen persönlichen Nähe zum Ordinarius als Ratgeber [...] an der bischöflichen Politik beteiligt waren." Bihrer 2014, 17-26.

4 Bihrer 2005, $559 \mathrm{f}$. 
Zum Augsburger Domkapitel liegen noch keine entsprechenden Forschungen vor. Deshalb ist das Augsburger Domkapitel auch in der Regionen übergreifenden Kirchenreformforschung kaum beachtet worden, allerdings fanden personengeschichtliche Bezüge Erwähnung: So war der Augsburger Domkustos Johannes Gwerlich auch Generalvikar des Bischofs Friedrich von Bamberg und als solcher 1427 in die Reform des Bamberger Michaelsklosters involviert. ${ }^{5}$ Für das Augsburger Domkapitel bedeutete dies freilich, dass Gwerlich hier kaum präsent sein konnte. Es ist nicht untypisch, dass sich der Bamberger Bischof in dieser Angelegenheit an seinen Generalvikar wandte. Als zwei Jahre später der Augsburger Bischof Peter von Schaumberg beginnend mit Benediktbeuren die Reform der Benediktinerklöster in seiner Diözese in Angriff nahm, bediente auch er sich für die Klostervisitation mit Johannes Kautsch seines Generalvikars. Diesem wurde noch der Wiener Theologieprofessor Johannes Hayterbach zur Seite gestellt. ${ }^{6}$ Weshalb nicht auch der reformerfahrene Domkapitular Gwerlich beteiligt wurde, ist nicht überliefert, angesichts seines Bamberger Amtes stand er aber für Aufgaben im Bistum Augsburg wohl nicht zur Verfügung. Kautsch war zu diesem Zeitpunkt noch nicht Mitglied des Augsburger Domkapitels, wurde in dieses aber ein Jahr später aufgenommen. ${ }^{7}$

Die genannten Beispiele zeigen uns bischöflich angeordnete Aktivitäten im Sinne der Melker Klosterreform. Die Mitgliedschaft im Augsburger Domkapitel spielte für die Auswahl der beauftragten Personen keine Rolle. Wichtiger dürfte das Amt des bischöflichen Generalvikars gewesen sein. Davon abgesehen achteten die Bischöfe auf die fachliche Kompetenz ihrer Reformkommissionen. Die jenseits dieser Auswahlkriterien bestehende oder bevorstehende Mitgliedschaft einzelner Reformbeauftragter im Augsburger Domkapitel illustriert aber, dass die Ideen der Melker Reform unter seinen Mitgliedern präsent waren. Als Institution hat sich das Augsburger Domkapitel damit aber nicht befasst, jedenfalls sind keine einschlägigen Kapitelsbeschlüsse überliefert.

Dieser Befund steht in einem gewissen Widerspruch zur Rolle des Domkapitels gemäß der kirchenverfassungsgeschichtlichen Tradition, wonach man annehmen könnte, dass sich wichtige bischöfliche Entscheidungen immer auch auf den Rat des Domkapitels zu stützen hatten. Diese Annahme entsprach allerdings

5 Siehe Studt 2004, 297 - hier, wohl nach Bamberger Quellen mit der Schreibweise „Gerlich“. In Augsburg sind die Schreibweisen Gwerlich, Guerlich und Werlich überliefert. Vgl. Haemmerle 1935, Nr. 285.

6 Hemmerle 1991, 168. - Studt 2004,186 f. (mit korrekturbedürftigen Angaben in Anm. 199).

7 Haemmerle 1935, Nr. 185. 
im 15. Jahrhundert nicht einmal der Erwartungshaltung des Augsburger Domkapitels. Diese finden wir in einem Dokument formuliert, dessen Berücksichtigung nach der rechtlich umstrittenen Inthronisation des Bischofs Anshelm von Nenningen (25. November 1413) quasi als nachträgliche,Wahlkapitulation` oder Herrschaftsvertrag von diesem am 7. Februar 1414 und zehn Jahre später wohl auch von seinem päpstlich providierten Nachfolger bestätigt wurde. ${ }^{8}$

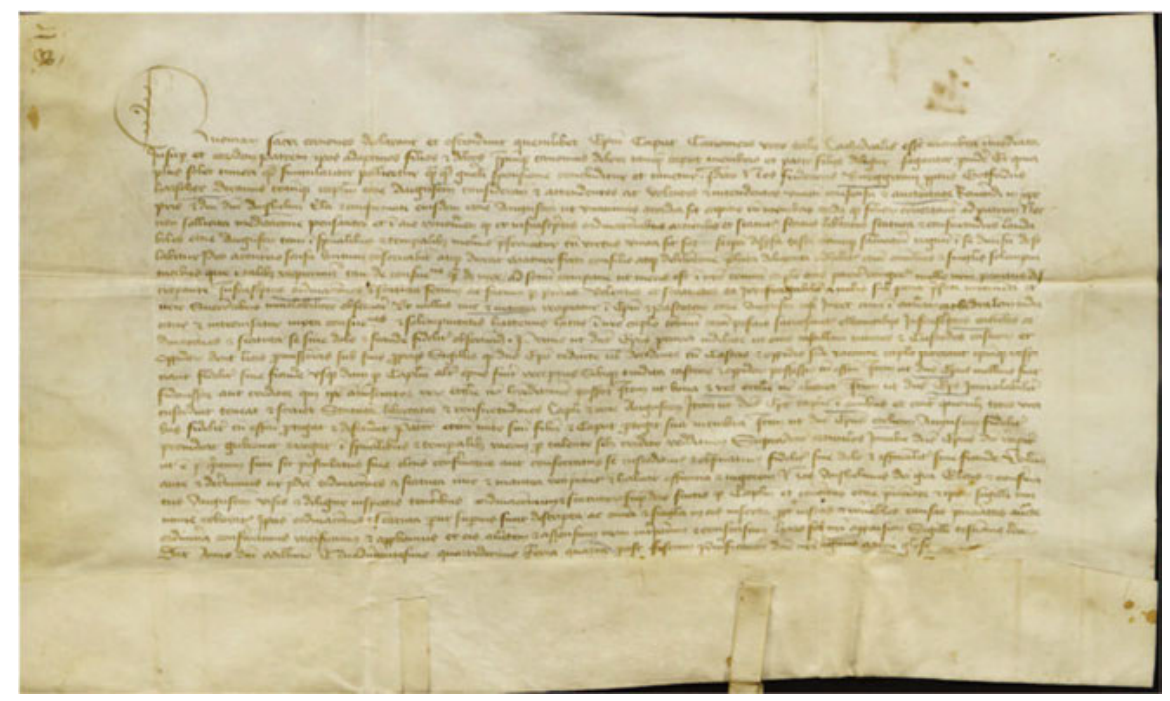

Abb. 1: Die Augsburger „Wahlkapitulation“vom 7. Februar 1414 (Foto: Staatsarchiv Augsburg).

Ein konkretes Mitspracherecht in Angelegenheiten der Diözesanleitung wurde hier vom Domkapitel nicht beansprucht. Das Augsburger Domkapitel wollte in Sedisvakanzen seine interimistische Kollegialhoheit über das Hochstift gesichert sehen und vom jeweils amtierenden Bischof gegen etwaige Gegner verteidigt werden. Darüber hinaus beschränkt sich das Dokument auf die allgemein gehaltenen Forderungen, wonach der Augsburger Bischof keine Güter entfremden, die „Statuten, Freiheiten und Gewohnheiten des Domkapitels und der Augsburger

8 Augsburg, Staatsarchiv, Domkapitel Urkunden Nr. 910 (Abbildung 1 - originalurkundliche Bestätigung Anshelms) und Münchner Bestand, Lit. 983, 207 f. (Abschrift der Bestätigung Bischof Peters). Druck in Monumenta Boica 34/1. München: Bayerische Akademie der Wissenschaften, 1844, 214-216, Nr. 101. Vgl. Krüger 2006, 46; Krüger 2013, 27; Schmidt 2015, 515 und 528 f. 
Kirche“ achten und insgesamt ein guter Bischof in spiritualibus et temporalibus sein solle. Die Domkapitulare seien dabei vom Bischof entsprechend alter kanonischer Bestimmungen wie „Adoptivsöhne“ oder eigene „Gliedmaßen“ anzusehen. Einschränkungen für die bischöfliche Regierung von Hochstift und Diözese konnten aus diesem Dokument im Unterschied zu manchen Wahlkapitulationen in anderen Bistümern des 15. Jahrhunderts nicht hergeleitet werden. ${ }^{9}$

\section{Geschichte und urkundliche Überlieferung}

Die Augsburger Wahlkapitulation führt uns zu der definitorischen Frage, was wir eigentlich unter dem Augsburger Domkapitel im 15. Jahrhundert zu verstehen haben. Offensichtlich gab es eine Diskrepanz zwischen dem, was ein Domkapitel seiner rechtsgeschichtlichen Tradition nach sein sollte und dem, was es in der politischen und sozialen Praxis des 15. Jahrhunderts leisten konnte. Das Domkapitel war das älteste kirchliche Kollegium in Augsburg, eine Gemeinschaft von Kanonikern der Kathedrale, die traditionell als Wahl- und Ratskolleg des Bischofs fungierte. Die Funktion als Wahlkolleg konnte es im 15. Jahrhundert aber nicht häufig ausüben: ${ }^{10}$ Die Postulation Anshelms von Nenningen durch die Mehrheit des Domkapitels am 14. August 1413 mündete in einen langjährigen Bistumsstreit, der erst 1424 mit der erfolgreichen päpstlichen Provision Peters von Schaumberg beigelegt wurde. Die lange Amtszeit Bischof Peters machte in den folgenden Jahrzehnten keine Wahl erforderlich. Noch zu seinen Lebzeiten wurde ihm mit Johann Graf von Werdenberg ein Koadjutor mit Nachfolgerecht zur Seite gestellt. In diese Vorgänge war das Domkapitel zwar involviert, dennoch kann erst nach Johanns Tod 1486 wieder von einer Bischofswahl die Rede sein. Diese Wahl, die das Pontifikat Friedrichs von Zollern (†1505) begründete, war die einzige erfolgreiche Bischofswahl durch das Domkapitel im gesamten 15. Jahrhundert.

Aber auch als Ratskolleg der Bischöfe konnte das Augsburger Domkapitel schon aus praktischen Gründen nicht regelmäßig in Erscheinung treten, denn die Bischöfe residierten zumeist in Dillingen, während das Kapitel seinen Sitz in

\footnotetext{
9 Deutlich konkreter waren etwa die Wahlkapitulationen in Würzburg und Bamberg - vgl. Krüger 2013, 249-252.

10 Vgl. hierzu und zum Folgenden die mit einschlägigen Quellenbelegen versehene Übersicht von Schmidt 2015, 837-841.
} 
Augsburg hatte. Darüber hinaus ist aber auch mit Absenzen zahlreicher Augsburger Domkapitulare an ihrem Kapitelssitz zu rechnen, wie das Beispiel der Bamberger Tätigkeit des Johannes Gwerlich gezeigt hat.

Der Forschungsstand zur Geschichte des Augsburger Domkapitels im 15. Jahrhundert ist noch deutlich erweiterungswürdig. Nach verschiedenen Arbeiten aus der ersten Hälfte des 20. Jahrhunderts ${ }^{11}$ sind zum mittelalterlichen Augsburger Domkapitel nur wenige Einzelaspekte, darunter für das 14. und 15. Jahrhundert vor allem das Verhältnis zur Augsburger Bürgerschaft, ${ }^{12}$ vertiefend untersucht worden. Weitere Analysen fehlen auch deshalb, weil der Stand der Quellenerschließung zum späteren Mittelalter als defizitär zu bezeichnen ist. Im Sommer 2014 hat deshalb im Staatsarchiv Augsburg und am Lehrstuhl für Mittelalterliche Geschichte der Universität Augsburg ein Erschließungsprojekt zum Urkundenbestand des Augsburger Domstiftsarchivs begonnen. Hier kann das 15. Jahrhundert allerdings zunächst nur in seinen Anfängen bis 1424 berücksichtigt werden. Nur vereinzelt wird der weitere Verlauf des Jahrhunderts schon gestreift, weil die Überlieferung der älteren Urkunden mitunter nur als Insert in jüngeren vorliegt. Außerdem wird ein Nachfolgeprojekt für die Zeit nach 1424 vorbereitet. Auf dieser Grundlage bestehen derzeit nur erste Einblicke in die urkundliche Überlieferung des 15. Jahrhunderts. Andere Quellengattungen sind teilweise schon besser erschlossen, können aber im vorliegenden Beitrag keine Berücksichtigung finden. ${ }^{13}$ Dieser stützt sich ergänzend zum Forschungsstand auf das Urkundenarchiv des Domkapitels.

Das Augsburger Domkapitelsarchiv war am Ende des alten Reiches in einen Kernbestand und kleinere ergänzende Bestände aufgeteilt, die bei der Säkularisation überwiegend nach München überführt wurden. Aus dem Kernbestand wurde allerdings ein kleinerer Teilbestand, vor allem zum Kapitelsbesitz in Schwäbisch-Gmünd und Lorch, extrahiert und entsprechend dem Grundsatz „Archiv folgt der Herrschaft“ nach Stuttgart gebracht. Diese Trennung konnte 1997 überwiegend rückgängig gemacht werden. Abgesehen von wenigen in Stuttgart

11 Grundlegend Leuze 1909, 1-114; Riedner 1909-1911, 43-90; Rückert 1916-1919, 183-254; Schöntag 1938.

12 Insbesondere im Rahmen der Dissertation von Kießling 1971, 323-352. Ergänzend mit Urkundenabbildungen Krüger 2012, 62-71.

13 Verwiesen sei hier besonders auf den Beitrag von Juliane Trede zur Augsburger Dombibliothek in diesem Band. Zu liturgiegeschichtlichen Quellen vgl. vorläufig Düring 1988, 32-46 und künftig die kurz vor dem Abschluss stehende Tübinger Dissertation von Jens Brückner. 
verbliebenen Stücken ${ }^{14}$ befinden sich heute alle bei der Säkularisation in Staatsbesitz übergegangenen Urkunden im Staatsarchiv Augsburg, wobei der zeitweilige, 165 Stücke umfassende Teilbestand Gmünd/Lorch hier derzeit noch als Sonderbestand aufbewahrt, im Zuge des laufenden Erschließungsprojektes aber wieder in den Kernbestand reintegriert werden wird. ${ }^{15}$ Bis 1804 umfasste dieser ohne Gmünd/Lorch und die Stuttgarter Einzelstücke - 8.628 Urkunden. Davon stammen die ältesten 800 Urkunden aus dem Zeitraum von 1099 bis 1400 . Aus der Zeit von 1400 bis 1499 stammen 2066 Urkunden. „Gmünd/Lorch“ und weitere Sonderbestände (Benefizien, Fraternitas Sancti Magni) dazu gerechnet, sind aus dem 15. Jahrhundert mehr als 2500 Urkunden erhalten. Durchschnittlich sind somit aus jedem Jahr des 15. Jahrhunderts ca. 25 Originalurkunden erhalten, die dem ehemaligen Domkapitelsarchiv zuzurechnen sind. Die Erschließung dieser Urkunden steht größtenteils noch aus. Die Inhalte dieser Urkunden sind jeweils einzeln und nur für sich betrachtet zu einem größeren Teil historisch nicht besonders interessant. Durch die Gesamterschließung in einer Datenbank bilden die Urkunden aber eine Grundlage, um ein umfassendes Bild von den persönlichen und besitzrechtlichen Verflechtungen des Domkapitels zu rekonstruieren und vielfältige Fragen zur Geschichte des 15. Jahrhunderts zu klären.

\section{Soziale Identität und Tätigkeitsschwerpunkte}

Zur Frage der sozialen Zusammensetzung und Identität des Augsburger Domkapitels wissen wir ohne diese künftige Forschungsgrundlage, dass es sich im 15. Jahrhundert erfolgreich dagegen gewehrt hat, Augsburger Bürgersöhne aufzunehmen. ${ }^{16}$ Schwieriger ist es, die Sozialgemeinschaft Domkapitel positiv zu definieren. Denn jenseits der Ausgrenzung Augsburger Bürgersöhne gab es keine so grundsätzlichen Ausschlusskriterien. Für die begehrten Pfründen kamen ade-

14 Dabei handelt es sich um Einzelstücke aus dem insgesamt nur 13 Urkunden umfassenden, teilweise aus dem ehemaligen Hochstiftsarchiv und teilweise aus dem Domkapitelsarchiv stammenden Bestand in Stuttgart, Hauptstaatsarchiv, A 130 a mit Betreffen zu württembergischen Besitzungen und Pfarreien, größerenteils zu Tannhausen (Ostalbkreis).

15 Eine die Pfarrei Gmünd, aber auch diejenige von Tannhausen betreffende Urkunde des Kardinals Pileo da Prata von 1379 (Bestätigung der Inkorporationen ins Augsburger Domkapitel) verblieb bei den genannten Einzelstücken im Hauptstaatsarchiv Stuttgart (Signatur A 130 a U13). Vgl. Hotz 2015, 69-95 und 161-171, hier besonders 71 mit Anm. 9.

16 Vgl. zuletzt Krüger 2014a, 38 f. 
lige, ritterbürtige oder promovierte Kleriker sowohl der Region als auch aus ferneren Gegenden in Betracht und es war de facto auch möglich, die Mitgliedschaft im Augsburger Domkapitel mit anderen einträglichen Ämtern in und außerhalb der Diözese Augsburg zu vereinen. ${ }^{17}$ Dies zeigt uns, dass wir mit einer Gemeinschaft von Pfründnern zu rechnen haben, die ihre Kanonikate mit individuell ganz unterschiedlichen Beziehungen nach Augsburg innehatten. Manche Domkapitulare haben sich jenseits des Bezugs ihrer Präbende für Augsburg nicht interessiert und sind dort auch nie präsent gewesen, so wie etwa am Ende des 15. Jahrhunderts der römische Adelige und Kardinal Giovanni Battista Savelli, der von 1487 bis 1497 sogar als Dompropst titulierte. ${ }^{18}$ Anders als in den meisten deutschen Domkapiteln war die Augsburger Dompropstei damals noch nicht aus dem Kapitel herausgelöst. Dies geschah aber am Ende des 15. Jahrhunderts, nachdem der Augsburger Bürgersohn (und nachmalige Salzburger Erzbischof und Kardinal) Mathäus Lang mit kaiserlicher Unterstützung seinen Anspruch auf diese Pfründe gegen den Kapitelswillen durchsetzen konnte. ${ }^{19}$

Theoretisch gab es für Inhaber der Augsburger Domkanonikate und Kapitelämter Präsenzpflichten in Augsburg. ${ }^{20}$ Das päpstliche Dispensrecht machte es aber möglich, dass diese Pflichten für einzelne Kapitelsmitglieder außer Kraft gesetzt waren. Liturgie und regelmäßige Chorgebete am Augsburger Dom waren davon kaum berührt, weil es hierfür ohnehin eine größere Zahl von Vikaren gab. Die Domkapitulare waren dagegen zwar ihrem Stand nach Kleriker, ihrem ausgeübten Beruf nach aber zu einem erheblichen Teil schlicht Vermögensverwalter.

Davon zeugt der überwiegende Anteil der ca. 2500 erhaltenen Urkunden aus dem 15. Jahrhundert. Angesichts eines umfangreichen, sich weiträumig erstreckenden, mit komplexen Verflechtungen und Rechtskonstruktionen verbundenen Immobilienbesitzes, der auch diverse gerichtliche, außergerichtliche und mitunter sogar gewalttätige Auseinandersetzungen implizierte, war das eine durchaus anspruchsvolle und zeitaufwändige Aufgabe. Das ist ein wesentlicher Grund dafür, dass Domkapitel eher Gegenstand von Reformdiskussionen als Impulsgeber für Reformen waren. Die Frage ist, ob sich das Augsburger Domkapitel in dieser Hinsicht von den Konventen anderer Stifte und Klöster unterschied. Die

17 Vgl. Krüger 2014b, $34 \mathrm{f}$.

18 Haemmerle 1935, 143, Nr. 706.

19 Leuze 1909, 52; Haemmerle 1935, 108 f., Nr. 530; Krüger 2013, 248 mit Anm. 80.

20 Die örtlichen Residenzbestimmungen seit 1297 erläutert Leuze 1909, 26-28. Es wurde versucht, diese dadurch zu unterstützen, dass ein Teil des Pfründenbezugs der Kanoniker als „Präsenzgelder“ in Abhängigkeit von der Beteiligung am regelmäßigen Chorgebet gezahlt wurde: Leuze 1909, 23-26. 
erhaltenen Urkundenbestände sprechen dafür, dass der Aufwand für die Vermögensverwaltung im Alltag sämtlicher Stifte und Klöster des 15. Jahrhunderts einen erheblichen Raum einnahm. Das gilt auch für solche Klöster, die wie St. Ulrich und Afra von der Melker Reform beeinflusst waren. ${ }^{21}$

Der wesentliche Unterschied zwischen Domkapiteln und Klöstern bestand im 15. Jahrhundert darin, dass die Klöster ausgehend von lokalen und regionalen Impulsen reformiert werden konnten. Die Melker Reform und die vielfältigen Initiativen zu ihrer Verbreitung sind hierfür anschauliche Beispiele. Die Verhältnisse in Domkapiteln waren dagegen stärker von universalkirchlichen Rahmenbedingungen abhängig, deren Reform zwar - besonders auf den großen Konzilien - das ganze 15. Jahrhundert über zur Diskussion stand, aber aufgrund zu vieler Detailprobleme und Interdependenzen nicht wirkungsvoll in Angriff genommen wurde.

Wenn wir in den Urkundenbeständen nach Beziehungen zwischen dem Domkapitel und St. Ulrich und Afra suchen, so finden wir in den Urkundenbeständen hierzu ausschließlich besitz- und vermögensrechtliche Aspekte. Die Verbindungen waren nicht besonders eng und ihre Belege sind nicht sehr zahlreich. Wiederholt finden wir die Nennung von St. Ulrich und Afra nicht als Kloster, sondern als Pfarreibezirk, weil das Domkapitel dort Häuser und Höfe besaß oder erwarb. Daraus ergaben sich keine substantiellen Beziehungen des Domkapitels zu Abt und Konvent des Klosters. Nur sehr selten können aus den überlieferten Urkunden auch Meinungsverschiedenheiten zwischen Domkapitel und Kloster über die Nutzungsrechte bei einzelnen Immobilien hergeleitet werden, so etwa aus einem Vergleichsvertrag vom 4. Januar 1501 bezüglich eines Ackers in Herbertshofen bei Meitingen. ${ }^{22}$

Die Äbte von Sankt Ulrich und Afra wurden vom Domkapitel gelegentlich zu Beglaubigungszwecken herangezogen: Abt Johann III. (Kissinger) von St. Ulrich

21 So umfasst etwa der ebenfalls besitzgeschichtlich dominierte Urkundenbestand von St. Ulrich und Afra schon für die ersten vier Jahrzehnte des 15. Jahrhunderts 422 erhaltene Stücke verzeichnet von Hipper 1956, 161-324, Nr. 410-831. Nach einem Urbar von 1446 besaß das Kloster Grundbesitz in 312 Siedlungen, so Liebhart 2011, 170.

22 Augsburg, Staatsarchiv, Domkapitel Augsburg Urkunden Nr. 2901. 
und Afra vidimierte am 20. August 1415 für das Domkapitel gleich fünf ältere Urkunden, ${ }^{23}$ Johann IV. am 27. September 1451 eine Urkunde des amtierenden Kaisers Friedrich III. ${ }^{24}$ und Johann V. am 30. April 1494 ebenfalls eine Urkunde Friedrichs III. ${ }^{25}$ Das Domkapitel nutzte in diesen Fällen die anscheinend in Stadt und Region Augsburg anerkannte Autorität der Äbte von Sankt Ulrich und Afra. Dies könnte darauf hindeuten, dass das bischöfliche Offizialat möglicherweise wegen zu großer persönlicher Nähe und daraus abgeleiteter Befangenheit für entsprechende Aufträge des Domkapitels nicht geeignet schien.

Aus der Zeit des späten 14. und frühen 15. Jahrhunderts können wir aus den von uns in der Datenbank bereits erfassten Urkunden einen Eindruck davon gewinnen, wie viele und welche Domherren zu dem in Augsburg präsenten Kern des Kapitels mit dokumentierter Geschäftstätigkeit gehörten. Die mit Abstand häufigsten urkundlichen Nachweise haben wir dabei von den Dompröpsten. Die Dekane sind nach dem bisherigen Befund seltener genannt, noch seltener die Scholaster, häufiger dagegen die Domkellerer. Insgesamt sprechen die Befunde dafür, dass wir von einem Kern von etwa 12 präsenten und geschäftstätigen Domherren ausgehen können. Das heißt, nur etwa die Hälfte der stimmberechtigten Kanoniker war mit dem Augsburger Domstift aktiv verbunden. ${ }^{26}$

Ungeachtet ihrer Schwerpunkte in der Vermögensverwaltung haben sich diese Domkapitulare Reformanliegen nicht völlig verschlossen. Die einzige bekannte Reformmaßnahme, die das Kapitel im 15. Jahrhundert für sich selbst durchführte, war aber die Heraufsetzung der Gelehrtenquote unter seinen Mitgliedern entsprechend dem Konkordat Martins V. mit der deutschen Nation auf dem Konstanzer Konzil. ${ }^{27}$ Demnach sollten mindestens ein Sechstel der Mitglieder einen Universitätsabschluss haben, wobei das Kapitel die Umsetzung so gestaltete, dass künftig bürgerliche Bewerber nur noch als Doktoren oder Lizenziaten einer höheren Fakultät aufgenommen wurden. Bei adeligen und ritterbürtigen Bewerbern genügte dagegen der Abstammungsnachweis. Das Kapitel erfüllte damit zwar die Gelehrtenquote, entsprach aber nicht ganz dem Geist der Konstanzer Reformbeschlüsse. Begründet wurde dies mit dem besonderen

23 Augsburg, Staatsarchiv, Domkapitel Augsburg Urkunden (DKAU) Nr. 918-922 mit Abschriften der vidimierten Originale mit den derzeitigen Nummern 129, 129a, 197, 222 und 306.

24 Augsburg, Staatsarchiv, Domkapitel Augsburg Urkunden Nr. 1244a.

25 Augsburg, Staatsarchiv, Domkapitel Augsburg Urkunden Nr. 2198.

26 Zur Zahl der Domkanonikate vgl. Leuze 1909, 6. Demnach schwankte die Zahl der stimmberechtigten Augsburger Domkapitulare im 15. Jahrhundert zwischen 22 und 27.

27 Augsburg, Staatsarchiv, Domkapitel Augsburg Urkunden 953, ed. in Monumenta Boica 34,1 1844, 274-276 Nr. 112. Die bezogene Konstanzer Bestimmung wurde zuletzt mit deutscher Übersetzung ediert von Miethke/Weinrich 1995, 518-521. 
Schutzbedürfnis für die Stiftsgüter. Tatsächlich nahm die Sozialgemeinschaft Domkapitel auch deutlich stärker einen Charakter als Rittergemeinschaft denn als Gelehrtengemeinschaft ein. ${ }^{28}$ Seit 1435 ist sogar eine förmliche Mitgliedschaft des Domkapitels im Ritterbund des St. Georgenschildes belegt. ${ }^{29}$ Andererseits steigerte sich dessen ungeachtet die Gelehrtenquote, da auch die adeligen und ritterbürtigen Mitglieder immer öfter Universitäten zumindest besucht hatten, wenn auch nicht immer mit einschlägigen Abschlüssen.

Das Augsburger Domkapitel war insofern sicherlich keine bildungsferne Gemeinschaft, und seine Mitglieder waren nicht generell reformfeindlich eingestellt. Die Reformimpulse, die das Kloster St. Ulrich und Afra und andere Klöster von der Diözesanleitung bekamen, gingen aber, soweit bislang erkennbar, nicht vom Domkapitel sondern von den Bischöfen aus. Besonders bei den Abtswahlen sind deutliche bischöfliche Einflussnahmen belegt, ohne dass eine Einbeziehung des Domkapitelsrates dabei greifbar würde, so etwa von Bischof Peter 1428 und 1457 in St. Ulrich und Afra ${ }^{30}$ und 1441 in Benediktbeuern ${ }^{31}$ oder von Bischof Johann 1479 in Ottobeuren. ${ }^{32}$ Wie auch die Klosterreformen fielen Fragen der Abtswahlen, soweit sich der Bischof nicht persönlich darum kümmerte, in die Zuständigkeit seines Generalvikars. ${ }^{33}$

\section{Konsens zur Reform}

Dies indiziert eine Professionalisierung der bischöflichen Diözesanverwaltung, in der regelmäßige Beratungen mit dem Domkapitel keine Rolle spielen konnten. In besitzrechtlichen Angelegenheiten blieb das förmliche Konsensrecht des Domkapitels allerdings intakt. Dafür spricht das Fehlen überlieferter Konflikte zwischen Bischof und Kapitel in Augsburg. Zu Recht wurde in der Forschung wiederholt die vergleichsweise gute Harmonie zwischen Bischof und Domkapitel in Augsburg festgestellt. ${ }^{34}$ Auch in den erhaltenen Urkunden ist der Konsens zwischen Bischof und Domkapitel immer wieder belegt. Dies kann zum Abschluss

28 Krüger 2014a, $38 \mathrm{f}$.

29 Uhl 1940, 118 f. mit Bezug auf diverse archivalische Überlieferungen ab 1435, wobei er den Beginn der Georgenschild-Mitgliedschaft des Kapitels schon früher vermutet.

30 Vgl. Unterburger 2011, 155 (zu 1428) und 156 (zu 1457).

31 Hemmerle 1991, 480.

32 Zoepfl 1955, 472.

33 Vgl. Hemmerle 1991, 474.

34 In diesem Sinne ausführlich Uhl 1940, 116-127. Vgl. Zoepfl 1955, 447 und 479. 
dieses Beitrages am Beispiel der Stiftungsurkunde des Bischofs Friedrich von Zollern für eine Domprädikatur illustriert werden. Wie der Beitrag von Werner Williams-Krapp in diesem Band zeigt, hat Bischof Friedrich den Wunsch zu einer solchen Stiftung, möglichst zu Gunsten seines Freundes Johannes Geiler von Kayersberg lange gehegt, ohne dieses Anliegen allerdings gegen den Willen des Augsburger Domkapitels durchzusetzen. Als Bischof Friedrich am 8. März 1505 in der Dillinger Residenz im Alter von 54 Jahren starb, befanden sich an seinem Sterbebett zwei Domherren. ${ }^{35}$ Das Domkapitel hatte nur wenige Wochen zuvor, seinen Konsens zu der Stiftungsprädikatur gegeben. Dieser Konsens wird in der erhaltenen Stiftungsurkunde vom 2. Januar 1505 sehr deutlich betont.

35 Zoepfl 1955, 534. 


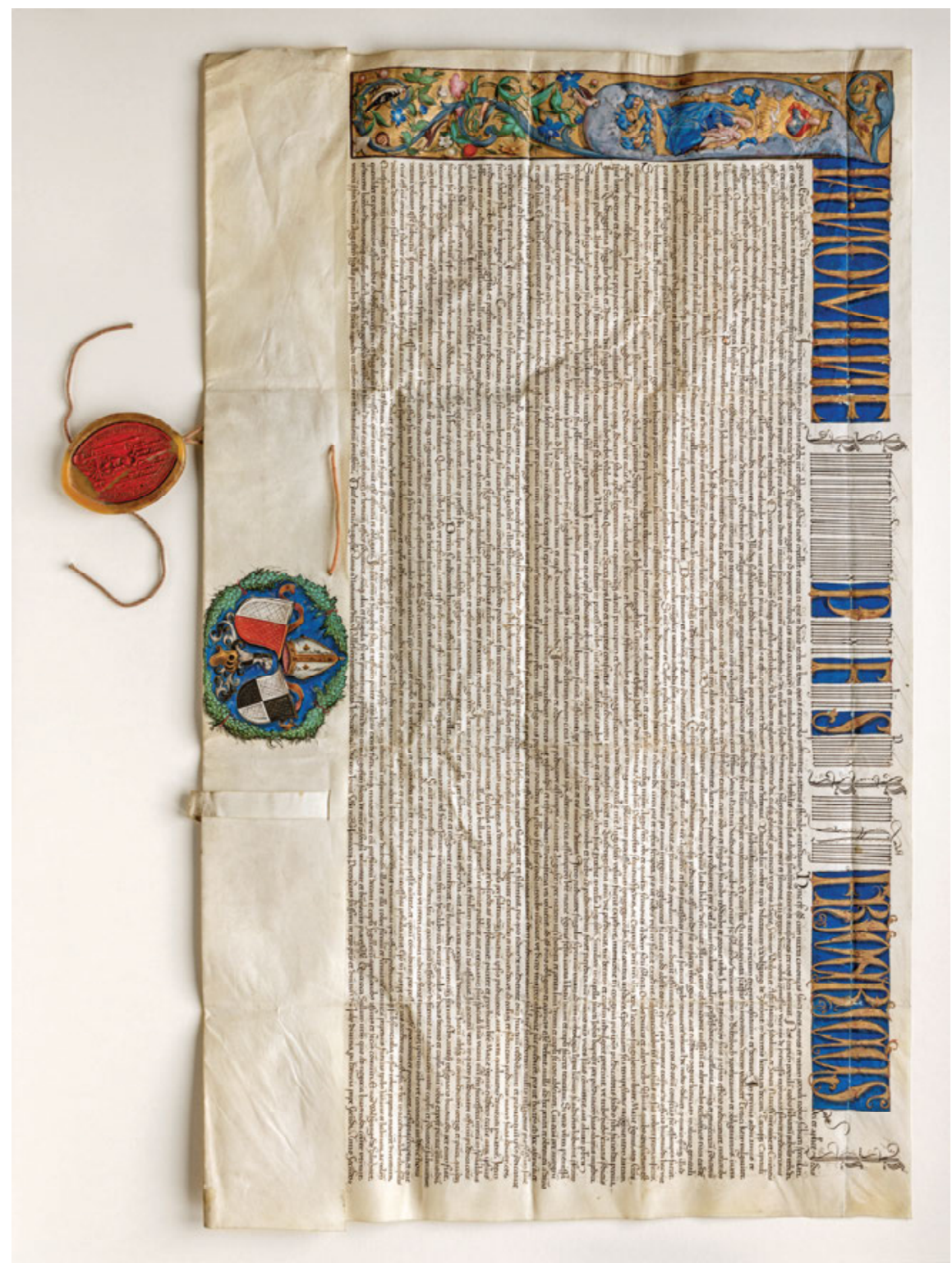

Abb. 2: Stiftungsurkunde der Augsburger Domprädikatur vom 2. Januar 1505 (Foto: Staatsarchiv Augsburg). 
Der Konsens galt sowohl der auf Dauer eingerichteten Prädikatur selbst als auch ihrer hohen Dotierung mit 100 rheinischen Gulden jährlich, die auch über den Tod Bischof Friedrichs hinaus aus dem bischöflichen Zehnten in Gremheim (Lkr. Dillingen) zu finanzieren war. Visuell fand der Konsens seinen Ausdruck in der Doppelbesiegelung der Urkunde durch Bischof und Domkapitel, die in der Corroboratio angekündigt und sehr wahrscheinlich auch ausgeführt wurde. Man mag es als symptomatisch interpretieren, dass das bischöfliche Siegel recht gut erhalten bis heute noch daran hängt, während das Domkapitelssiegel verloren ist: abgesehen von geringen Wachsspuren hat es sich komplett von der noch bestehenden Pergamentpressel gelöst. Derartige Phänomene sind allerdings in mittelalterlichen Urkundenbeständen keine Seltenheit, und als wahrscheinlichste Gründe müssen hierfür fast immer äußere Zufälle gelten.

Das abgefallene Kapitelssiegel ist also ein eher gewöhnlicher Aspekt dieser Urkunde. Aufmerksamkeit verdient sie vielmehr wegen ihrer sonstigen Ausführung. Es handelt sich um eine in der Forschung bisher noch unbeachtete großformatige, illuminierte Prachturkunde. Die Miniaturen könnten in der Augsburger Werkstatt des Leonhard Beck entstanden sein. ${ }^{36}$ Die nicht unbeträchtlichen Kosten für diese Ausführung dürfte wohl der ausstellende Bischof alleine getragen haben. Da sie die Verhältnisse am Dom und damit das Domkapitel betraf, ging sie aber nach der Ausfertigung in den Besitz des Domkapitels über und kann als ein beeindruckendes Zeugnis der dortigen Renaissancekultur gelten. Neben der hohen künstlerischen Qualität der Miniaturen ist auch das originell eingesetzte Repertoire verschiedener Schriftarten bemerkenswert, das Anklänge an historische Urkundenschriften erkennen lässt, ohne insgesamt ein bestimmtes Vorbild zu kopieren. Es ist davon auszugehen, dass eine Prachturkunde mit solchen Elementen auch im Umfeld des Reformklosters St. Ulrich und Afra mit einer gewissen Begeisterung aufgenommen worden wäre. Dort entstand wohl nur wenige Jahre später das Schriftmusterbuch von Leonhard Wagner, in dem das Interesse an historischen Schriften nochmals deutlich ausgeprägter dokumentiert wird. ${ }^{37}$

Das Urkundenarchiv des Augsburger Domkapitels führt uns damit im kursorischen Durchgang vom Beginn des 15. bis zum Anfang des 16. Jahrhunderts letzt-

36 So Juliane Trede bei der Diskussion des Tagungsbeitrages und bei einer späteren Führung für eine Augsburger Universitätsgruppe. Vgl. ihren Beitrag zur Augsburger Dombibliothek in diesem Band. Aus der Dombibliothek weist etwa Clm 3895 ähnlich geartete Miniaturen auf, die der Beck-Werkstatt zugeschrieben werden. Vgl. auch Augsburg, Staats- und Stadtbibliothek, Cod. 49a, f. 165v, abgebildet in Pfändtner 2010: http://www.historisches-lexikon-bayerns.de/Lexikon/Buchmalerei (Spätmittelalter) [30. Juli 2016].

37 Abbildung und Beschreibung: Miedaner 1993, 71 f., Nr. 50. 
lich zu einer Reformmaßnahme am Augsburger Dom in Verbindung mit dem Ausdruck farbenfroher, kunstsinniger und gelehrter Kultur. Allerdings sind wir zu diesem Beispiel nur in so kurzer Zeit gekommen, weil wir ca. 2500 Beurkundungen besitz- und vermögensrechtlicher Betreffe weithin unbeachtet gelassen haben. Die Prädikatur-Stiftungsurkunde von 1505 muss im Gesamtkontext als exzeptionell bezeichnet werden. Ungeachtet des beurkundeten Konsenses ist die Urkunde sicherlich kein Ausdruck eines Reformeifers im spätmittelalterlichen Augsburger Domkapitel. Andererseits spricht die Urkunde aber auch nicht für Reformresistenz. Die überwiegend ritterbürtigen Augsburger Domkapitulare waren mit der allgemeinen Kultur- und Ideengeschichte ihrer Zeit verflochten. Ihre Tätigkeitsschwerpunkte lagen aber auf dem Gebiet der Vermögensverwaltung. 


\section{Literatur}

Bihrer, Andreas: Der Konstanzer Bischofshof im 14. Jahrhundert. Herrschaftliche, soziale und kommunikative Aspekte. Ostfildern 2005.

Bihrer, Andreas: „Die Konstanzer Bischofswahlen im 14. Jahrhundert. Zu den Gruppenbildungen am Bischofshof und im Domkapitel“, in: Rottenburger Jahrbuch für Kirchengeschichte 33 (2014): 17-26.

Düring, Walter: „Zur Geschichte der Augsburger Domliturgie im Mittelalter“, in: Jahrbuch des Vereins für Augsburger Bistumsgeschichte 22 (1988): 32-46.

Fouquet, Gerhard: Das Speyerer Domkapitel im späten Mittelalter (ca. 1350-1540). Adlige Freundschaft, fürstliche Patronage und päpstliche Klientel. Mainz 1987.

Haemmerle, Albert: Die Canoniker des Hohen Domstiftes zu Augsburg bis zur Saecularisation. Zürich 1935.

Hemmerle, Josef: Die Benediktinerabtei Benediktbeuern. Berlin/New York 1991.

Hipper, Richard: Die Urkunden des Reichsstiftes St. Ulrich und Afra in Augsburg 1023-1440. Augsburg 1956.

Holbach, Rudolf: Stiftsgeistlichkeit im Spannungsfeld von Kirche und Welt. Studien zur Geschichte des Trierer Domkapitels und Domklerus im Spätmittelalter. 2 Teilbände. Trier 1982.

Hollmann, Michael: Das Mainzer Domkapitel im späten Mittelalter 1306-1478. Mainz 1990.

Hotz, Brigitte: „Zwischen Kirchenspaltung und Konzilsidee: Ein Urkundenbündel des Kardinals Pileo da Prata für das Augsburger Domkapitel von 1379“, in: Suevia et Ecclesia. Festgabe für Georg Kreuzer zum 75. Geburtstag. Sonderdruck aus der Zeitschrift des Historischen Vereins für Schwaben (mit einem erweiterten Abbildungsteil), hrsg. von Thomas M. Krüger und Christof Paulus. Augsburg 2015. 69-95 und 161-171.

Kießling, Rolf: Bürgerliche Gesellschaft und Kirche in Augsburg im Spätmittelalter. Ein Beitrag zur Strukturanalyse der oberdeutschen Reichsstadt. Augsburg 1971.

Krüger, Thomas M.: „Die Hausherren des Doms und sein funktionsgeschichtlicher Wandel: Bischof und Domkapitel im mittelalterlichen Augsburg“, in: Der Augsburger Dom im Mittelalter, hrsg. von Martin Kaufhold. Augsburg 2006. 27-48.

Krüger, Thomas M.: „Gewalt und Recht. Bürgerlich-klerikale Streitkultur im mittelalterlichen Augsburg“, in: Städtische Kultur im Mittelalterlichen Augsburg, hrsg. von Martin Kaufhold. Augsburg 2012. 62-71.

Krüger, Thomas M.: Leitungsgewalt und Kollegialität. Vom benediktinischen Beratungsrecht zum Konstitutionalismus deutscher Domkapitel und des Kardinalkollegs. Berlin/Boston 2013.

Krüger 2014a = Krüger, Thomas M.: „Korporative Identitätsentwicklung des Augsburger Domkapitels im Spiegel seiner mittelalterlichen Statuten“, in: Rottenburger Jahrbuch für Kirchengeschichte 33 (2014): 27-40.

Krüger 2014 b = Krüger, Thomas M.: „Motive und Probleme mittelalterlicher Kleriker- und Religiosenmigration. Beispiele aus dem Bistum Augsburg im europäischen Vergleich“, in: Mobilität und Migration in der Region, hrsg. von Reinhard Baumann und Rolf Kießling. Konstanz und München 2014. 23-44.

Leuze, Otto: „Das Augsburger Domkapitel im Mittelalter“, in: Zeitschrift des Historischen Vereins für Schwaben 35 (1909): 1-114. 
Liebhart, Wilhelm: „Großgrundbesitz, Verwaltung und Haushalt. Zur Wirtschaftsgeschichte von St. Ulrich und Afra im Spätmittelalter und in der Frühen Neuzeit“, in: Benediktinerabtei St. Ulrich und Afra in Augsburg (1012-2012). Geschichte, Kunst, Wirtschaft und Kultur einer ehemaligen Reichsabtei. Festschrift zum tausendjährigen Jubiläum, hrsg. von Manfred Weitlauff. Lindenberg 2011. 166-179.

Miedaner, Stefan: „Proba Centum scripturarum, Schriftmusterbuch von Leonhard Wagner, zwischen 1507 und 1517“, in: Aus zwölfJahrhunderten Augsburger Bistumsgeschichte. Katalog zur Ausstellung des Bistumsarchivs Augsburg anläßlich des 64. Deutschen Archivtags vom 27.-30. September 1993 in Augsburg, hrsg. von Stefan Miedaner. Augsburg 1993. 71 f., Nr. 50.

Miethke, Jürgen/Weinrich, Lorenz: Quellen zur Kirchenreform im Zeitalter der großen Konzilien des 15. Jahrhunderts. Band 1: Die Konzilien von Pisa (1409) und Konstanz (1414-1418). Darmstadt 1995.

Monumenta Boica 34/1. München 1844.

Riedner, Otto: „Besitzungen und Einkünfte des Augsburger Domkapitels um 1300“, in: Archiv für die Geschichte des Hochstifts Augsburg 1 (1909-1911): 43-90.

Rückert, Georg: „Die Präbende am Domkapitel zu Augsburg“, in: Archiv für die Geschichte des Hochstifts Augsburg 5 (1916-1919): 183-254.

Schmidt, Andreas: „Bischof bist Du und Fürst“, in: Die Erhebung geistlicher Reichsfürsten im Spätmittelalter - Trier, Bamberg, Augsburg. Heidelberg 2015.

Schöntag, Ilse: Untersuchungen über die persönliche Zusammensetzung des Augsburger Domkapitels im Mittelalter. Zeulenroda 1938.

Studt, Birgit: Papst Martin V. (1417-1431) und die Kirchenreform in Deutschland. Köln/Weimar/Wien 2004.

Uhl, Anton: Peter von Schaumberg. Kardinal und Bischof von Augsburg 1424-1469. Ein Beitrag zur Geschichte des Reiches, Schwabens und Augsburgs im 15. Jahrhundert. Speyer 1940.

Unterburger, Klaus: „Zwischen freier Reichsstadt und monastischer Reform. Leben und Gelehrsamkeit in St. Ulrich und Afra im 15. Jahrhundert.", in: Benediktinerabtei St. Ulrich und Afra in Augsburg (1012-2012). Geschichte, Kunst, Wirtschaft und Kultur einer ehemaligen Reichsabtei. Festschrift zum tausendjährigen Jubiläum, hrsg. von Manfred Weitlauff. Lindenberg 2011. 147-165.

Zoepfl, Friedrich: Das Bistum Augsburg und seine Bischöfe im Mittelalter. München/Augsburg 1955. 